\title{
Role of the caudal pressor area in the regulation of sympathetic vasomotor tone
}

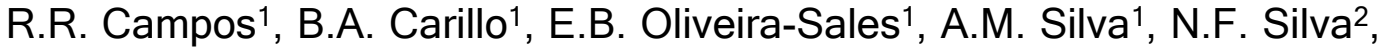 \\ H.A. Futuro Neto ${ }^{3,4}$ and C.T. Bergamaschi ${ }^{5}$ \\ 1Departamento de Fisiologia, Disciplina de Fisiologia Cardiovascular e Respiratória, Escola Paulista de \\ Medicina, Universidade Federal de São Paulo, São Paulo, SP, Brasil \\ ${ }^{2}$ Laboratório de Neuromorfologia, ${ }^{3}$ Programa de Pós-Graduação em Ciências Fisiológicas, Universidade \\ Federal do Espírito Santo, Vitória, ES, Brasil \\ ${ }^{4}$ Escola Superior de Ciências, Santa Casa de Misericórdia de Vitória (EMESCAM), Vitória, ES, Brasil \\ ${ }^{5}$ Departamento de Biociênicas, Universidade Federal de São Paulo, Campus Baixada Santista, Santos, \\ SP, Brasil
}

Correspondence to: R.R. Campos, Disciplina de Fisiologia Cardiovascular e Respiratória, EPM, UNIFESP, Rua Botucatu, 862, 04023-060 São Paulo, SP, Brasil

Fax: +55-11-5573-7820. E-mail: campos@fcr.epm.br

\begin{abstract}
It is well known that the ventrolateral medulla contains neurons involved in the tonic and reflex control of the cardiovascular system. Two regions within the ventrolateral medulla were initially identified: the rostral ventrolateral medulla (RVLM) and the caudal ventrolateral medulla (CVLM). Activation of the RVLM raises arterial blood pressure and sympathetic nerve activity, and activation of the CVLM causes opposite effects. The RVLM premotor neurons project directly to sympathetic preganglionic neurons and are involved in the maintenance of resting sympathetic vasomotor tone. A significant proportion of tonic activity in the RVLM sympathetic premotor neurons is driven by neurons located in a third region of the ventrolateral medulla denominated caudal pressor area (CPA). The CPA is a pressor region located at the extreme caudal part of the ventrolateral medulla that appears to have an important role controlling the activity of RVLM neurons. In this brief review, we will address the importance of the ventrolateral medulla neurons for the generation of resting sympathetic tone related to arterial blood pressure control focusing on two regions, the RVLM and the CPA.
\end{abstract}

Key words: Rostral ventrolateral medulla; Rat; Glutamate; Blood pressure; Caudal pressor area; Sympathetic vasomotor tone

Publication supported by FAPESP.

Received April 24, 2007. Accepted July 4, 2008

\section{Introduction}

Sympathetic vasomotor outflow is composed of efferent pathways that control vascular tone (vascular peripheral resistance) and cardiac output (contractility and heart rate), playing an essential role in maintaining the constancy of blood pressure and bodily homeostasis. The motor outflow of the sympathetic nervous system originates predominantly from the intermediolateral cell column in preganglionic neurons that are topographically organized in the spinal cord; they are the last neuronal link for the peripheral activation of sympathetic post-ganglionic neurons and adrenal medulla cells. An interesting characteristic of the barosensitive vasomotor sympathetic fibers is that they have tonic activity (sympathetic tone). This tonic activity of sympathetic fibers is probably restricted to sympathetic neurons involved with cardiovascular functions (vasoconstriction and cardioaccelerator) (1).

Sympathetic vasomotor tone and arterial pressure depend critically on the tonic activity of a restricted group of spinal projecting neurons, the sympathetic premotor neurons. Direct projections to the intermediolateral column originate predominantly from at least five areas of the brain: a) rostral ventrolateral medulla (RVLM), b) rostral 
ventromedial medulla, c) caudal raphe nuclei, d) A5 cell group in the pons, and e) paraventricular nucleus of the hypothalamus (PVN) (2). Of these five nuclei, the PVN and the RVLM appear to have a tonic effect on the control of sympathetic vasomotor tone and are topographically organized (2-5).

In this brief review, we address the question of the importance of ventrolateral medulla neurons in the generation of the sympathetic vasomotor tone focusing on two regions, the RVLM and the caudal pressor area (CPA). The CPA is a pressor region located at the extreme caudal part of the caudal ventrolateral medulla (CVLM), which appears to have an important role in directly or indirectly controlling the activity of RVLM neurons.

\section{Rostral ventrolateral medulla}

The RVLM receives input from a number of different nuclei in the brain and also sends projections to many other regions involved in cardiovascular, respiratory and hormonal control. Reciprocal innervations between the RVLM and other brain nuclei strongly suggest that the RVLM is not only an important region involved in maintaining tonic sympathetic vasomotor tone, but it is also an integrative center controlling cardiovascular functions, processing information from peripheral nerves (baroreceptor, chemoreceptor and somatosympathetic reflexes) and from other brain nuclei. After processing information, the RVLM affects the sympathetic outflow and the release of specific hormones adjusting the cardiovascular system during behavioral states such as exercise, sleep and defense reaction. The RVLM premotor neurons can selectively control the sympathetic outflow to individual tissues and this feature is probably important for the central organization of specific autonomic responses $(4,6)$.

The RVLM neurons have been studied extensively using different approaches. First, they were identified by topical application of drugs (glycine and leptazol) on the ventral surface of the brainstem in cats. When applied to the rostral part of the ventrolateral medulla, glycine caused a large fall in blood pressure, in the region denominated as the glycinesensitive area, which is another denomination for the RVLM (7). In contrast, stimulation of these neurons by leptazol caused an increase in blood pressure (8). Subsequently, the anatomical location of the RVLM was defined first in the rabbit (9) and subsequently in the rat and cat $(10,11)$. A more precise localization of the RVLM neurons was obtained by microinjecting amino acids (glutamate and gamma-aminobutyric acid (GABA)) directly into the ventrolateral medulla parenchyma. The cardiovascular neurons were then located ventral to the rostral part of the nucleus ambiguous and caudal to the end pole of the facial nucleus.

RVLM neurons have been studied and characterized using extracellular microelectrode recording (12-15) and intracellular microelectrodes (16). However, despite the large number of studies on the RVLM premotor neurons and their recognized importance for the control of the sympathetic vasomotor tone, the basis for the ongoing tonic activity of these neurons is not yet fully understood. There are two major hypotheses regarding the activity of the RVLM neurons: one is that the RVLM presympathetic neurons have pacemaker activity (17) and the other is the "network hypothesis", which suggests that these neurons are driven by others in the brainstem (18).

The "pacemaker hypothesis" proposed by Guyenet (17) was based on studies using intracellular recording of the RVLM in medullary slices and states that the ongoing activity of the basal RVLM is determined by intrinsic autodepolarization of the premotor neuron. According to this hypothesis, synaptic inputs are only a modulator factor on the intrinsic activity generated by the presympathetic RVLM neurons. However, apparently the pacemaker pattern has been demonstrated only in in vitro preparations. In in vivo conditions, the pacemaker pattern has yet to be demonstrated.

Under specific experimental conditions in vivo, fast excitatory synaptic inputs appear to drive the RVLM spiking activity. The ongoing activity of the RVLM neurons resulted from synaptic inputs, with individual action potential usually preceded by identifiable fast excitatory synaptic inputs (16). At least under these experimental conditions, no pacemaker activity was found in the RVLM. This finding is consistent with the "network hypothesis" for the generation of sympathetic vasomotor tone proposed by Barman and Gebber (18). This hypothesis states that the activity of premotor neurons, in vivo, depends on excitatory inputs from other brainstem nuclei acting as part of an oscillating network. However, the components of this network are not completely understood and a question that remains to be answered is: Where is the source or sources of the synaptic drive to the RVLM?

There is a large body of evidence identifying a number of nuclei in the brain and peripheral nerves from which synaptic excitation of RVLM neurons can be achieved following electrical or chemical stimulation (1). Although a number of brainstem regions, when activated, cause sympathetic activation via RVLM, few regions may provide a tonic excitatory drive to the RVLM neurons to support their activity. It is well known that the PVN, the pontine reticular formation and the CPA provide a tonic drive to the RVLM (15). Our hypothesis in this brief review is that the CPA is a major source of tonic excitatory drive to the RVLM. 


\section{Caudal pressor area}

The CPA was discovered in cats by Feldberg and Guertzenstein (19). They described a region in the cat under deep anesthesia with chloralose that was located caudal to the depressor region of the CVLM that presented the same characteristics as the RVLM. Stimulation and inhibition of this region caused an increase and a decrease in blood pressure, respectively. The authors suggested that the CPA appears to be responsible for maintaining arterial blood pressure and sympathetic vasomotor tone during deep anesthesia. Later, Gordon and McCann (20) described the same region in urethane-anesthetized rats in which glutamate microinjection into the CPA caused an increase in blood pressure. An interesting finding described by Gordon and McCann was that the cardiovascular responses elicited by the CPA were mediated by the RVLM.

In anesthetized rats, bilateral inhibition of the CPA by GABA or glycine decreases blood pressure by $30-40 \mathrm{mmHg}$ (21). In rabbits, CPA inhibition caused a similar decrease in arterial pressure and almost abolished renal sympathetic nerve activity (22). Furthermore, bilateral microinjections of kynurenic acid into the CPA reduced the pressor response to stimulation of nucleus raphe obscurus (23). These data suggest that the CPA is important for maintaining the sympathetic vasomotor tone.

In conscious rats, unilateral L-glutamate microinjections into the CPA caused a dose-dependent increase in arterial pressure and respiratory rate. These responses were markedly attenuated by subsequent urethane anesthesia of the same animal (24).

The fall in arterial pressure during CPA inactivation is in part mediated by a decrease in RVLM activity. It was shown in barosensitive and reticulospinal RVLM sympathetic premotor neurons that there is an inhibition of approximately $40 \%$ in the firing rate of RVLM neurons during unilateral or bilateral microinjection of glycine into the CPA (15). The low-frequency RVLM neurons were the most affected. It is possible that the faster firing RVLM neurons receive more excitatory drive from sources other than the $\mathrm{CPA}$, such as the paraventricular nucleus of the hypothalamus, and this may contribute to their higher resting activity.

The location of the CPA in the medulla is less precise than the RVLM and was defined by Sun and Panneton (25), using glutamate microinjections to map the region. They defined that the CPA neurons are located lateral to the caudal end of the lateral reticular nucleus and ventromedial to the medullary dorsal horn near the level of the pyramidal decussation. They also showed that CPA sends projections to the RVLM and to the CVLM. The anatomical projections from the CPA to other nuclei in the ventrolateral medulla probably mediate the cardiovascular actions from this region. In fact, blockade of glutamatergic and GABAergic synapses within the CVLM impairs cardiovascular responses to CPA inhibition and stimulation, respectively $(21,26)$. Another possible mechanism was proposed by Natarajan and Morrison (27), who suggested that the pathway from CPA to RVLM involves glutamatergic activation within the CVLM. A possible pathway from CPA to other brainstem nuclei cannot the excluded, projections to pontine $A 5$ cells and to the RVLM can also be involved in the cardiovascular responses mediated by the CPA. Recently, Carolina Takakura et al. (28) reported that the excitatory responses mediated by the CPA are in part dependent on the commissural nucleus of the tractus solitarius. Therefore, it is possible that the commissural nucleus of the tractus solitarius is part of the circuitry activated by the CPA to induce cardiovascular responses. Figure 1 shows the possible pathways by which CPA neurons could control sympathetic vasomotor sympathetic activity.

A projection from the CPA to the preganglionic neurons in the spinal cord was recently described; however, if this cell group has any direct influence on sympathetic vaso-

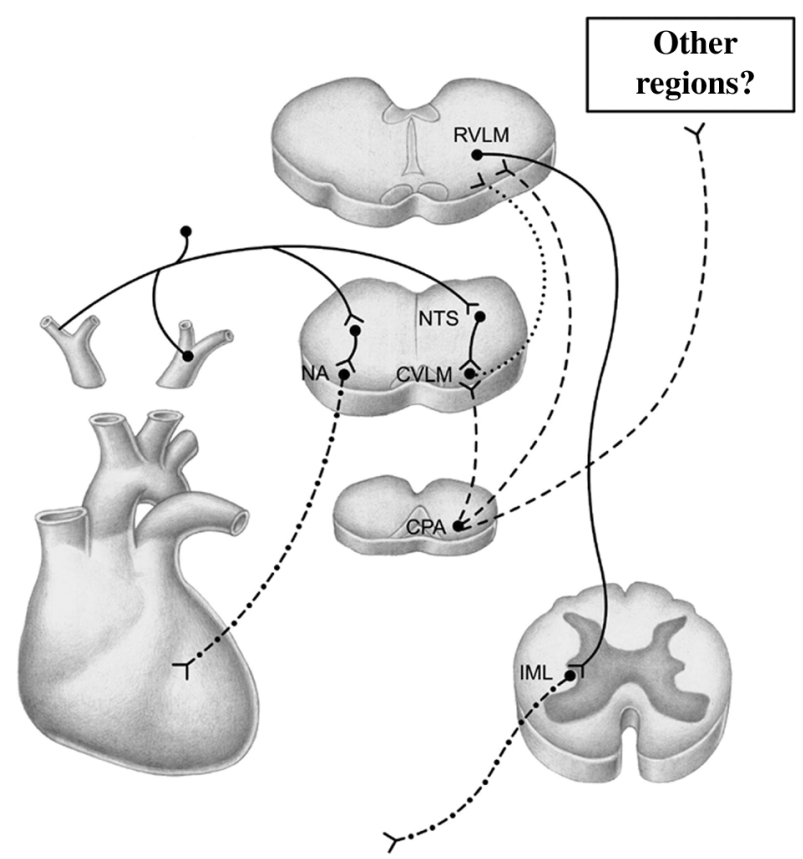

Figure 1. Schematic representation of pathways by which the caudal pressor area (CPA) can interfere with the sympathetic vasomotor tone. RVLM = rostral ventrolateral medulla; $\mathrm{CVLM}=$ caudal ventrolateral medulla; NTS $=$ nucleus of the tractus solitarius; $\mathrm{IML}=$ intermediolateral column; $\mathrm{NA}=$ nucleus ambiguus. Dotted line = GABAergic projection; continuous line = glutamatergic projection; dashed line $=$ modulatory projections; dash-dot line $=$ cholinergic projections . 
motor tone, acting as a sympathetic premotor group, remains to be determined (29). Apparently, the actions of the CPA depend on the RVLM, inhibition of the RVLM blocks the cardiovascular responses mediated by the CPA. However, inhibition of the CPA does not block the cardiovascular actions mediated by the RVLM $(20,21)$. These data suggest that there is probably a hierarchy between these two regions, with a dominant role of the RVLM.

CPA activation by glutamate causes a large increase in the renal sympathetic nerve activity, compared to that in response to RVLM stimulation. We showed that the sympathetic responses mediated by the CPA are not different in magnitude from those induced by the RVLM. However, the action of the CPA on sympathetic drive has weaker baroreceptor modulation compared to the responses mediated by the RVLM (30).

The effects of CPA inhibition on ongoing sympathetic nerve activity were first reported by Horiuchi and Dampney (31) in rabbits. They described a dramatic fall in renal sympathetic nerve activity in response to CPA inhibition, an effect similar to that observed during RVLM inhibition.
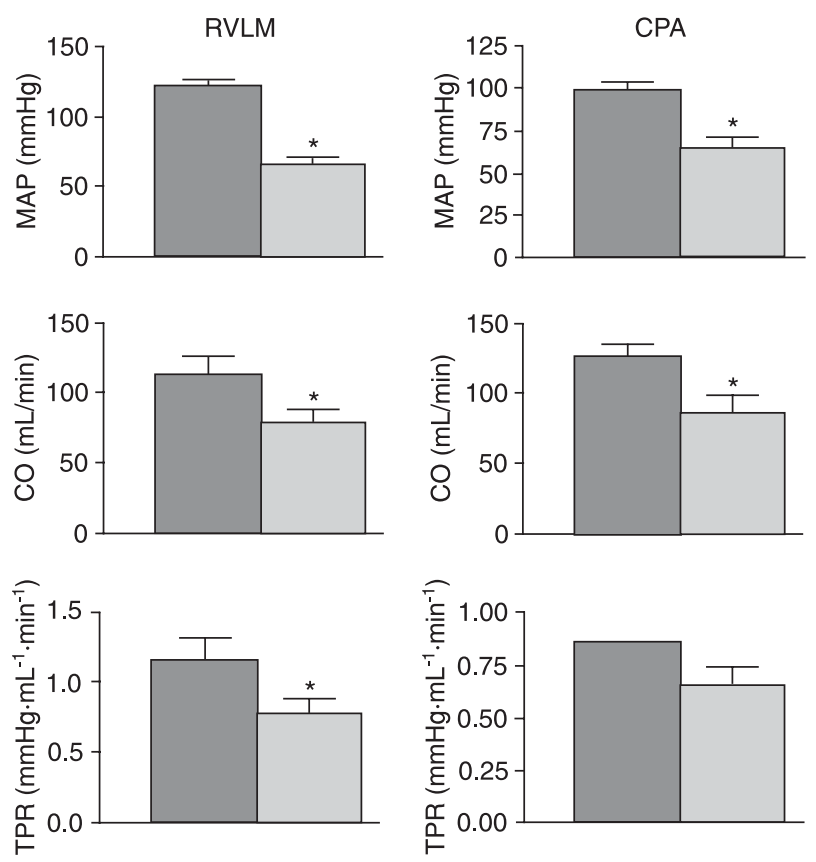

Figure 2. Effect of GABA microinjection into the rostral ventrolateral medulla (RVLM) or into the caudal pressor area (CPA). GABA, $50 \mathrm{nmol} / 100 \mathrm{~nL}$ bilaterally into 6 rats for each region. Data are reported as means \pm SEM. Rats were anesthetized with urethane $(1.2 \mathrm{~g} / \mathrm{kg}, i \mathrm{v})$. Dark gray columns = basal conditions; light gray columns $=$ experimental conditions. MAP = mean arterial pressure; $\mathrm{CO}=$ cardiac output; TPR $=$ total peripheral resistance. ${ }^{*} \mathrm{P}<0.05$ compared to basal conditions (unpaired Student $t$-test)
These data indicate that this region can modify sympathetic nerve activity (excitatory and inhibitory actions) as much as the RVLM.

The decrease in blood pressure in response to CPA inhibition by GABA microinjection was mainly due to a decrease in cardiac output, there was a non-statistically significant trend to a decrease in the vascular resistance, as shown in Figure 2. However, during inhibition of the RVLM there was a significant decrease in both, cardiac output and in the total peripheral resistance (Figure 2). Despite the fact that the cardiovascular responses mediated by the CPA and RVLM are similar, the physiological role of the CPA is not well defined. An action in the physiology of nociception was proposed (32). However, the possibility that the CPA is part of the rostral continuation of a population of cervical neurons that can control sympathetic nerve activity (33) or part of the lateral CVLM cannot be excluded and remains to be elucidated.

More recently, a novel pressor area at the medullocervical junction was described (34). Chemical stimulation of a region extending from the most caudal ventrolateral medulla into the upper cervical spinal cord evoked large sympathetically mediated pressor responses. This response was not dependent on the integrity of the RVLM and the region was denoted as the medullo-cervical pressor area (MCPA). This region is probably distinct from the CPA and the role of this novel descending sympathoexcitatory region in central cardiovascular regulation remains to be elucidated.

Taken together, these data suggest that an important fraction of resting activity of sympathetic premotor neurons of the RVLM depends on a synaptic drive from the CPA. However, there is no information on what drives the CPA neurons.

\section{Perspectives}

It is well known that the ventrolateral medulla contains the principal premotor neurons involved in the modulation of sympathetic vasomotor tone and arterial blood pressure. There is increasing evidence to support the view that increased sympathetic nerve activity plays an important role in the pathogenesis of arterial hypertension (35). Therefore, changes in the excitatory and/or inhibitory neurotransmission within the ventrolateral medulla might constitute a mechanism that is essential for the development and maintenance of hypertension. The mechanisms by which the RVLM and the sympathoexcitatory neurons located in the caudal end of the ventrolateral medulla (CPA and MCPA) interact to control the sympathetic vasomotor tone in normotensive and hypertensive subjects remain to be determined. 


\section{References}

1. Guyenet PG. The sympathetic control of blood pressure. Nat Rev Neurosci 2006; 7: 335-346.

2. Strack AM, Sawyer WB, Hughes JH, Platt KB, Loewy AD. A general pattern of CNS innervation of the sympathetic outflow demonstrated by transneuronal pseudorabies viral infections. Brain Res 1989; 491: 156-162.

3. Dampney RA. Functional organization of central pathways regulating the cardiovascular system. Physiol Rev 1994; 74: 323-364.

4. McAllen RM, May CN, Campos RR. The supply of vasomotor drive to individual classes of sympathetic neuron. Clin Exp Hypertens 1997; 19: 607-618.

5. Allen AM. Inhibition of the hypothalamic paraventricular nucleus in spontaneously hypertensive rats dramatically reduces sympathetic vasomotor tone. Hypertension 2002; 39: 275-280.

6. Morrison SF. Differential control of sympathetic outflow. Am J Physiol Regul Integr Comp Physiol 2001; 281: R683R698.

7. Guertzenstein PG, Silver A. Fall in blood pressure produced from discrete regions of the ventral surface of the medulla by glycine and lesions. J Physiol 1974; 242: 489-503.

8. Feldberg W, Guertzenstein PG. Vasodepressor effects obtained by drugs acting on the ventral surface of the brain stem. J Physiol 1976; 258: 337-355.

9. Dampney RA. Brain stem mechanisms in the control of arterial pressure. Clin Exp Hypertens 1981; 3: 379-391.

10. Ross CA, Ruggiero DA, Park DH, Joh $T H$, Sved AF, Fernandez-Pardal $\mathrm{J}$, et al. Tonic vasomotor control by the rostral ventrolateral medulla: effect of electrical or chemical stimulation of the area containing $\mathrm{C} 1$ adrenaline neurons on arterial pressure, heart rate, and plasma catecholamines and vasopressin. J Neurosci 1984; 4: 474-494.

11. McAllen RM. Action and specificity of ventral medullary vasopressor neurones in the cat. Neuroscience 1986; 18: 51-59.

12. Brown DL, Guyenet PG. Cardiovascular neurons of brain stem with projections to spinal cord. Am J Physiol 1984; 247: R1009-R1016.

13. Morrison SF, Milner TA, Reis DJ. Reticulospinal vasomotor neurons of the rat rostral ventrolateral medulla: relationship to sympathetic nerve activity and the $\mathrm{C} 1$ adrenergic cell group. J Neurosci 1988; 8: 1286-1301.

14. Terui N, Saeki $\mathrm{Y}$, Kumada M. Barosensory neurons in the ventrolateral medulla in rabbits and their responses to various afferent inputs from peripheral and central sources. Jpn $J$ Physiol 1986; 36: 1141-1164.

15. Campos RR, McAllen RM. Tonic drive to sympathetic premotor neurons of rostral ventrolateral medulla from caudal pressor area neurons. Am J Physiol 1999; 276: R1209R1213.

16. Lipski J, Kanjhan R, Kruszewska B, Rong W. Properties of presympathetic neurones in the rostral ventrolateral medulla in the rat: an intracellular study "in vivo". J Physiol 1996; 490 (Part 3): 729-744.

17. Guyenet PG. Role of ventral medulla oblongata in blood pressure regulation. In: Loewy AD, Spyer KM (Editors), Central regulation of autonomic functions. New York: Oxford University Press; 1990. p 145-267.
18. Barman SM, Gebber GL. Lateral tegmental field neurons of cat medulla: a source of basal activity of ventrolateral medullospinal sympathoexcitatory neurons. J Neurophysiol 1987; 57: 1410-1424.

19. Feldberg W, Guertzenstein PG. Blood pressure effects of leptazol applied to the ventral surface of the brain stem of cats. J Physiol 1986; 372: 445-456.

20. Gordon FJ, McCann LA. Pressor responses evoked by microinjections of L-glutamate into the caudal ventrolateral medulla of the rat. Brain Res 1988; 457: 251-258.

21. Campos RR Jr, Possas OS, Cravo SL, Lopes OU, Guertzenstein PG. The caudal pressor area: A new region of the ventrolateral medulla involved in cardiovascular regulation. In: Trouth $\mathrm{O}$, Millis RM, Kiwull-Schone $\mathrm{H}$, Schlafke ME (Editors), Lung biology in health and disease. Vol. 82. New York: Marcel Dekker Inc.; 1995. p 817-835.

22. Dampney RA, Horiuchi J, Tagawa T, Fontes MA, Potts PD, Polson JW. Medullary and supramedullary mechanisms regulating sympathetic vasomotor tone. Acta Physiol Scand 2003; 177: 209-218.

23. Silva NF, Pires JG, Dantas MA, Futuro Neto HA. Excitatory amino acid receptor blockade within the caudal pressor area and rostral ventrolateral medulla alters cardiovascular responses to nucleus raphe obscurus stimulation in rats. Braz J Med Biol Res 2002; 35: 1237-1245.

24. Silva NF, Pires JG, Campos RR, Futuro Neto HA. Cardiovascular and respiratory responses to microinjection of Lglutamate into the caudal pressor area in conscious and anesthetized rats. Braz J Med Biol Res 2001; 34: 16031606.

25. Sun W, Panneton WM. The caudal pressor area of the rat: its precise location and projections to the ventrolateral medulla. Am J Physiol Regul Integr Comp Physiol 2002; 283: R768-R778.

26. Campos Junior RR, Possas OS, Cravo SL, Lopes OU, Guertzenstein PG. Putative pathways involved in cardiovascular responses evoked from the caudal pressor area. Braz J Med Biol Res 1994; 27: 2467-2479.

27. Natarajan M, Morrison SF. Sympathoexcitatory CVLM neurons mediate responses to caudal pressor area stimulation. Am J Physiol Regul Integr Comp Physiol 2000; 279: R364R374.

28. Carolina Takakura A, Santos Moreira T, Menani JV, Ribeiro Campos R, Colombari E. Commissural nucleus of the solitary tract is important for cardiovascular responses to caudal pressor area activation. Brain Res 2007; 1161: 32-37.

29. Li Q, Goodchild AK, Seyedabadi M, Pilowsky PM. Preprotachykinin A mRNA is colocalized with tyrosine hydroxylase-immunoreactivity in bulbospinal neurons. Neuroscience 2005; 136: 205-216.

30. de Toledo Bergamaschi C, de Arruda Carillo B, Futuro Neto HA, Campos RR. Differential baroreceptor modulation mediated by the ventrolateral medulla. Auton Neurosci 2006; 126-127: 156-162.

31. Horiuchi J, Dampney RA. Evidence for tonic disinhibition of RVLM sympathoexcitatory neurons from the caudal pressor area. Auton Neurosci 2002; 99: 102-110.

32. Sun W, Panneton WM. Defining projections from the caudal pressor area of the caudal ventrolateral medulla. J Comp 
Neurol 2005; 482: 273-293.

33. Jansen AS, Loewy AD. Neurons lying in the white matter of the upper cervical spinal cord project to the intermediolateral cell column. Neuroscience 1997; 77: 889-898.

34. Seyedabadi M, Li Q, Padley JR, Pilowsky PM, Goodchild
AK. A novel pressor area at the medullo-cervical junction that is not dependent on the RVLM: efferent pathways and chemical mediators. J Neurosci 2006; 26: 5420-5427.

35. Esler M. The sympathetic system and hypertension. Am J Hypertens 2000; 13: 99S-105S. 